\title{
A quantum-mechanical anharmonic oscillator with a most interesting spectrum
}

\author{
Paolo Amore ${ }^{a}$, Francisco M. Fernández ${ }^{\text {b,* }}$ \\ a Facultad de Ciencias, Universidad de Colima, Bernal Díaz del Castillo 340, Colima, Colima, Mexico \\ b INIFTA (CONICET), División Química Teórica, Blvd. 113 y 64(S/N), Sucursal 4, Casilla de Correo 16, 1900 \\ La Plata, Argentina
}

\section{H I G H L I G H T S}

- A quasi-exactly solvable anharmonic oscillator that depends on a parameter.

- The perturbation series for the lowest eigenvalue converges for positive values of the parameter.

- The perturbation series for the eigenfunction and expectation values are divergent.

- The perturbation series are Padé and Borel-Padé summable for positive values of the parameter.

- For negative values of the parameter there is an infinite number of avoided crossings.

\section{A R T I C L E I N F O}

\section{Article history:}

Received 5 May 2017

Accepted 15 July 2017

Available online 27 July 2017

\section{Keywords:}

Anharmonic oscillator

Quasi-exactly solvable

Perturbation theory

Padé summation

Avoided crossings

\begin{abstract}
A B S T R A C T
We revisit the problem posed by an anharmonic oscillator with a potential given by a polynomial function of the coordinate of degree six that depends on a parameter $\lambda$. The ground state can be obtained exactly and its energy $E_{0}=1$ is independent of $\lambda$. This solution is valid only for $\lambda>0$ because the eigenfunction is not square integrable otherwise. Here we show that the perturbation series for the expectation values are Padé and Borel-Padé summable for $\lambda>0$. When $\lambda<0$ the spectrum exhibits an infinite number of avoided crossings at each of which the eigenfunctions undergo dramatic changes in their spatial distribution that we analyse by means of the expectation values $\left\langle x^{2}\right\rangle$.
\end{abstract}

(c) 2017 Elsevier Inc. All rights reserved.

\footnotetext{
* Corresponding author. E-mail addresses: paolo.amore@gmail.com (P. Amore), fernande@quimica.unlp.edu.ar (F.M. Fernández).
} 


\section{Introduction}

Some time ago Herbst and Simon [1] discussed some interesting and baffling features of two onedimensional Hamiltonians. In one of them, $H^{(2)}(g)=p^{2}+x^{2}-1+g^{4} x^{6}+2 g^{2} x^{4}-3 g^{2} x^{2}$, the exact ground-state energy is $E^{(2)}(g)=0$ and the coefficients of the perturbation series $\sum a_{n}^{(2)} g^{2 n}$ vanish for all $n>0$. However, the perturbation series for the eigenvector $\Omega^{(2)}(g)$ is divergent at least in the norm sense. The related oscillator $H^{(3)}(g)=H^{(2)}($ ig $)$ is most interesting because $0<E^{(3)}(g)<D^{\prime} \exp \left(-c / g^{2}\right)$. Its potential has three wells and there is a kind of asymptotic degeneracy of expected states.

Those models are particular cases of the so-called quasi-exactly solvable Schrödinger equations [2] (and references therein). In fact, Turbiner [2] chose the closely related potential $V_{0}(x ; a, b)=a^{2} x^{6}+$ $2 a b x^{4}+\left(b^{2}-3 a\right) x^{2}-b$ for the discussion of the most interesting problem of phase transition.

The purpose of this paper is the analysis of the spectra of $H^{(2)}$ and $H^{(3)}$ because they exhibit several interesting features that may not emerge so clearly from the remarkable theoretical analysis carried out by Herbst and Simon [1] and Turbiner [2]. Present results are shown in Section 2 and conclusions in Section 3.

\section{The model}

For simplicity, here we rewrite the Hamiltonian proposed by Herbst and Simon [1] as

$$
H(\lambda)=H^{(2)}(2 \sqrt{\lambda})+1=p^{2}+x^{2}-12 \lambda x^{2}+8 \lambda x^{4}+16 \lambda^{2} x^{6} .
$$

It exhibits an exact ground-state eigenfunction

$$
\varphi(x)=\exp \left(-x^{2} / 2-\lambda x^{4}\right)
$$

with eigenvalue $E_{0}=1$. This solution is only valid for $\lambda \geq 0$ because it is not square integrable for negative values of $\lambda$.

In principle, one expects the eigenfunctions and eigenvalues of $H(\lambda)$ to have perturbation expansions about $\lambda=0$ of the form

$$
\begin{aligned}
\psi_{n}(x) & =\sum_{p=0}^{\infty} \psi_{n}^{(p)}(x) \lambda^{p}, \\
E_{n} & =\sum_{p=0}^{\infty} E_{n}^{(p)} \lambda^{p} .
\end{aligned}
$$

For the normalized ground-state eigenfunction we have

$$
\begin{aligned}
\psi_{0}(x) & =\frac{\varphi(x)}{\sqrt{\langle\varphi \mid \varphi\rangle}} \\
& =\frac{1}{\sqrt{2} \pi^{1 / 4}} e^{-x^{2} / 2}\left[1+\frac{1}{4}\left(3-4 x^{4}\right) \lambda+\frac{1}{32}\left(16 x^{8}-24 x^{4}-183\right) \lambda^{2}+\cdots\right],
\end{aligned}
$$

but all the perturbation corrections of the corresponding eigenvalue vanish $\left(E_{0}^{(j)}=0, j>0\right)$ as mentioned above. Therefore, perturbation theory fails to provide suitable values of $E_{0}(\lambda)$ when $\lambda<0$. The reason is that this eigenvalue behaves asymptotically as [1]

$$
E_{0}(\lambda)-1 \approx A|\lambda|^{B} e^{-C /|\lambda|}, \lambda<0 .
$$

Fig. 1 shows that $E_{0}(\lambda)-1$ already behaves in this way. A straightforward least-squares fitting for sufficiently small values of $|\lambda|$ suggests that $A \approx 0.891, B=0$ (as argued by Herbst and Simon [1]) and $C=1 / 8$.

Before proceeding any further with the more interesting properties of this fascinating model we want to point out that it is by no means unique. In fact, there are many exactly solvable models that 


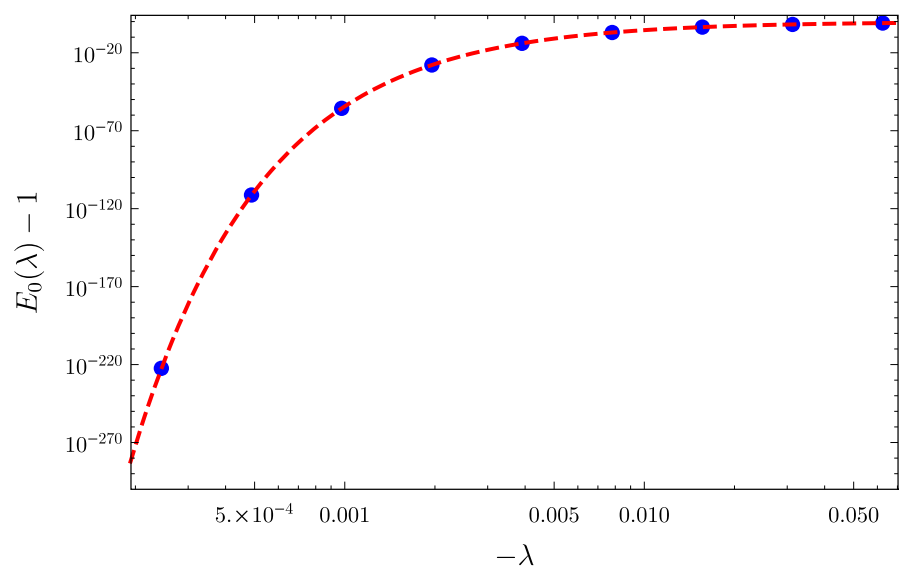

Fig. 1. $E_{0}(\lambda)-1$ calculated by means of the Rayleigh-Ritz variational method (dashed red line) and its least-square fitting using Eq. (5) (blue points).

exhibit a similar behaviour. For example, choose a quadratically-integrable function $\varphi(x)$ and define $W(x)=\varphi(x)^{-1} \varphi^{\prime}(x)$. The function $\varphi(x)$ is solution to the differential equation $\left[-\frac{d^{2}}{d x^{2}}+Q(x)\right] \varphi(x)=0$, where $Q=W^{\prime}+W^{2}$. If $W$ is a polynomial function of a parameter $\lambda, W(x)=W_{0}(x)+W_{1}(x) \lambda+$ $\cdots+W_{k}(x) \lambda^{k}$, then $Q(x)=Q_{0}(x)+Q_{1}(x) \lambda+\cdots+Q_{2 k}(x) \lambda^{2 k}$. Although these two polynomials have finite degrees the eigenfunction exhibits an infinite $\lambda$-power series given by the expansion of $\varphi(x)=\exp \left[\int_{x_{0}}^{x} W(t) d t\right]$. If we write $Q(x)=V(x)-E$, then the eigenvalue $E$ will be a polynomial function of $\lambda$ of degree at most $2 k$. Therefore, we see that there are many quantum mechanical models that exhibit an eigenfunction with an infinite perturbation series and the corresponding eigenvalue with just a finite number of perturbation corrections.

As additional examples consider $\varphi(x)=x \exp \left(-x^{2} / 2-\lambda x^{4}\right)$ that is the first excited state of the anharmonic oscillator with potential $V(x)=x^{2}+4 \lambda x^{2}\left(2 x^{2}-5\right)+16 \lambda^{2} x^{6}$ with eigenvalue $E_{1}=3$ and $\varphi(x)=\exp \left(-x^{2} / 2-\lambda x^{2} / 2-\lambda x^{4}\right)$ that is eigenfunction of the anharmonic oscillator with potential $V(x)=x^{2}+x^{2}\left(\lambda^{2}-10 \lambda\right)+8 \lambda x^{4}(\lambda+1)+16 \lambda^{2} x^{6}$ and eigenvalue $E_{0}=1+\lambda$. In the latter case the perturbation expansion for the eigenvalue has two terms. In this simple and straightforward way one can build as many examples as desired.

Although the perturbation series for the lowest eigenvalue of the oscillator (1) converges for all $\lambda$ the perturbation series for its eigenfunction is divergent [1]. As an illustrative example consider the expectation value

$$
\left\langle x^{2}\right\rangle=\frac{1}{2}-3 \lambda+48 \lambda^{2}-1188 \lambda^{3}+39168 \lambda^{4}-1604448 \lambda^{5}+\cdots .
$$

for the ground state. In what follows we resort to the notation $X_{n}=\left\langle x^{2 n}\right\rangle$ and $X_{n}^{(j)}$ for the perturbation correction of order $j$. We can easily calculate the perturbation corrections of $E_{n}^{(j)}$ and $X_{n}^{(j)}$ analytically to any desired order by means of the hypervirial perturbation method [3]. This approach is an example of perturbation theory without wavefunction that is based on the hypervirial relations and the Hellmann-Feynman theorem. If $\psi$ is an eigenfunction of the Hamiltonian $H$ with eigenvalue $E$ and $O$ is a linear operator, then $\langle\psi|[H, O]| \psi\rangle=0$ is called an hypervirial equation. If $H=-\frac{d^{2}}{d x^{2}}+V(x)$, where $V(x)$ is a polynomial function of $x^{2}$, the hypervirial relations for $O=O_{n}=-\frac{2 n+1}{2} x^{2 n}+x^{2 n+1} \frac{d}{d x}$, $n=0,1, \ldots$ yield a recurrence relation for the expectation values $X_{n}$. This recurrence relation that contains the energy $E$ as a coefficient of one of the terms is insufficient to solve the problem. The Hellmann-Feynman theorem $\frac{d E}{d \lambda}=\left\langle\frac{\partial V}{\partial \lambda}\right\rangle$ provides an additional equation that enables us to obtain all the perturbation corrections $X_{n}^{(j)}$ and $E^{(j)}$ in terms of $E^{(0)}$. 


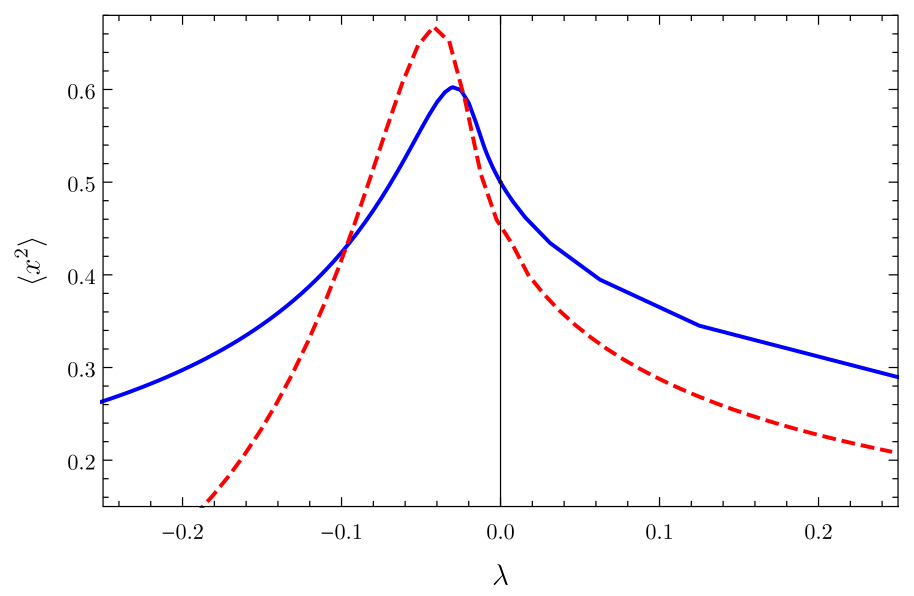

Fig. 2. Numerical $\left\langle x^{2}\right\rangle$ (blue, continuous line) and $\Re S_{B}(\lambda)$ (dashed, red line) for the ground state of the oscillator (1).

A least-squares fitting of the first 1000 perturbation coefficients enables us to estimate the asymptotic expansion

$$
X_{1}^{(j)}=(-1)^{j} 8^{j} j !\left[f_{0}+\frac{f_{1}}{j+1}+\frac{f_{2}}{(j+1)^{2}}+\cdots\right], j \gg 1,
$$

where

$$
\begin{aligned}
& f_{0}=0.450158158079, f_{1}=-0.168809309279, f_{2}=-0.305966873069 \\
& f_{3}=-0.869104178243, f_{4}=-3.78728795807, f_{5}=-22.6102214156
\end{aligned}
$$

On keeping just the leading term $X_{1}^{(j)} \sim f_{0}(-1)^{j} 8^{j} j$ ! the Borel sum yields

$$
\begin{aligned}
S(\lambda) & =f_{0} \sum_{j=0}^{\infty}(-1)^{j}(8 \lambda)^{j} j !=f_{0} \int_{0}^{\infty} e^{-t} \sum_{j=0}^{\infty}(-1)^{j}(8 \lambda t)^{j} \\
S_{B}(\lambda) & =f_{0} \int_{0}^{\infty} \frac{e^{-t}}{1+8 \lambda t} d t=f_{0} \frac{e^{\frac{1}{8 \lambda}}}{8 \lambda}\left[\operatorname{Shi}\left(\frac{1}{8 \lambda}\right)-\operatorname{Chi}\left(\frac{1}{8 \lambda}\right)\right],
\end{aligned}
$$

where

$$
\operatorname{Shi}(x)=\int_{0}^{x} \frac{\sinh t}{t} d t, \quad \operatorname{Chi}(x)=\int_{0}^{x} \frac{\cosh t}{t} d t .
$$

The Borel sum $S_{B}(\lambda)$ is complex for $\lambda<0$ and

$$
\Im S_{B}(\lambda) \sim 0.176715|\lambda|^{-1} e^{-\frac{1}{8 \lambda}}, \quad \lambda \rightarrow 0^{-} .
$$

Fig. 2 shows that the real part of $S_{B}(\lambda)$ exhibits a maximum for $\lambda<0$ like the actual value of $\left\langle x^{2}\right\rangle$.

The perturbation series originated in the expansion of a potential about one of its minima can be shown to be non-Borel summable when the potential has degenerate minima [4]. It has been argued that in such a case the imaginary part of the Borel sum is cancelled by the imaginary part of a logarithmic term [4]. In the present case the perturbation series are Padé and Borel-Pade summable for $\lambda>0$ as shown in Fig. 3 for $\left\langle x^{2}\right\rangle$ (ground state). This figure shows that the Borel summation improves the accuracy of the Padé approximant $[6 / 6](\lambda)$. However, both summation methods fail for $\lambda<0$. 


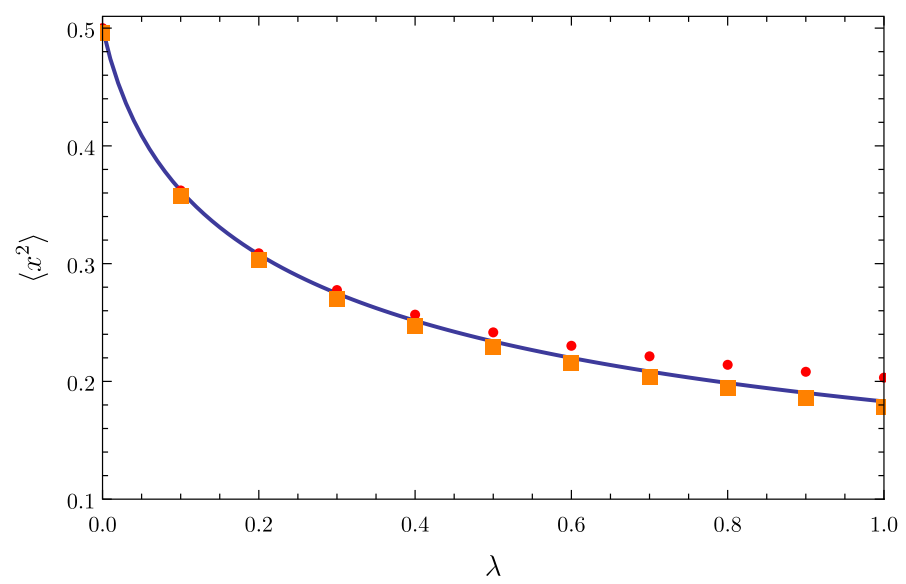

Fig. 3. Exact $\left\langle x^{2}\right\rangle$ (solid line) for the ground state of the oscillator (1) and the [6/6] Padé (circles) and Borel-Padé (squares) sums of the perturbation series.

The perturbation series for the excited states

$$
\begin{aligned}
& E_{1}(\lambda)=3+12 \lambda-144 \lambda^{2}+4176 \lambda^{3}-172800 \lambda^{4}+8892288 \lambda^{5}+\cdots \\
& E_{2}(\lambda)=5+48 \lambda-864 \lambda^{2}+36864 \lambda^{3}-2194560 \lambda^{4}+158810112 \lambda^{5}+\cdots,
\end{aligned}
$$

are divergent; for example

$$
E_{1}^{(j)} \sim(-1)^{j+1} \sqrt{j} 8^{j} j !
$$

was also obtained by numerical least-squares fitting of the analytical perturbation corrections calculated by means of the hypervirial perturbation method [3].

The divergence of the perturbation series for the eigenvalues and eigenfunctions of anharmonic oscillators is well understood. The reason is the existence of sequences of branch points in the complex $\lambda$ plane that approach the origin and lead to a zero radius of convergence $[5,6]$.

Fig. 4 shows the energy spectrum for small negative values of $\lambda$. In order to understand its structure we should pay attention to the form of the potential-energy function. When $0<\lambda<1 / 12$ the potential is a single well and becomes a double well when $\lambda>1 / 12$, but these cases are not relevant for present discussion and we just mention them for completeness. When $\lambda<-1 / 36$ the potential is a single well; when $-1 / 36<\lambda<0$ it exhibits three wells, one of them at the origin and the other two at $\pm x_{m}$, where

$$
x_{m}^{2}=-\frac{\sqrt{36 \lambda+1}+2}{12 \lambda}=-\frac{1}{4 \lambda}-\frac{3}{2}+\frac{27 \lambda}{2}-243 \lambda^{2}+\cdots
$$

These side wells are separated from the central one by two barriers located at $\pm x_{M}$ where

$$
x_{M}^{2}=\frac{\sqrt{36 \lambda+1}-2}{12 \lambda}=-\frac{1}{12 \lambda}+\frac{3}{2}-\frac{27 \lambda}{2}+243 \lambda^{2}+\cdots
$$

Clearly the side wells move away from the origin as $\lambda \rightarrow 0^{-}$. The values of the potential at these stationary points are $V(0)=0$,

$$
\begin{aligned}
& V\left(x_{m}\right)=\frac{(\sqrt{36 \lambda+1}+2)(\sqrt{36 \lambda+1}+36 \lambda-1)}{54 \lambda}=3+9 \lambda-54 \lambda^{2}+\cdots, \\
& V\left(x_{M}\right)=\frac{(\sqrt{36 \lambda+1}-2)(\sqrt{36 \lambda+1}-36 \lambda+1)}{54 \lambda}=-\frac{1}{27 \lambda}+1-9 \lambda+\cdots .
\end{aligned}
$$




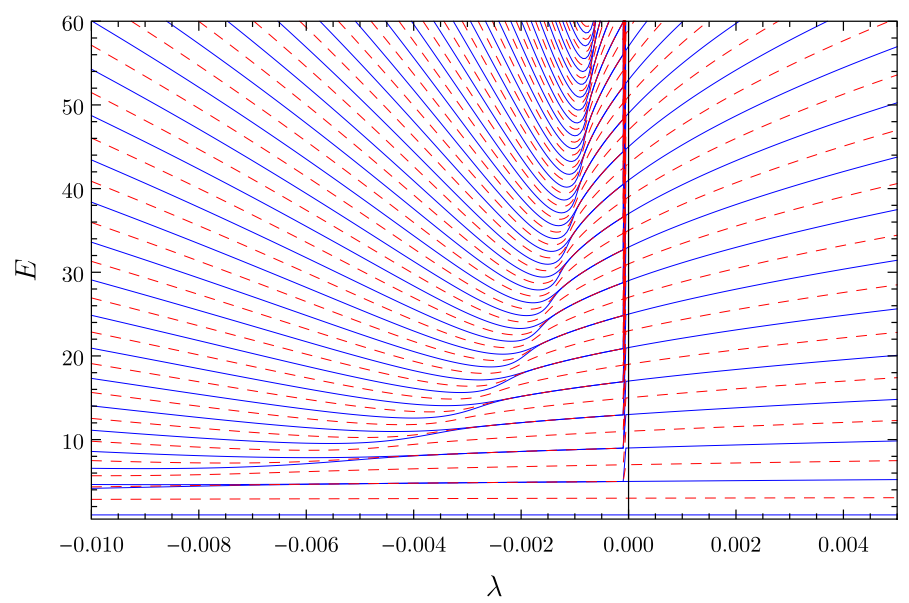

Fig. 4. Part of the spectrum of the anharmonic oscillator (1). Even and odd states are denoted by continuous (blue) and dashed (red) lines, respectively.

Note that the minima are bounded from below while the maxima increase unboundedly. In the limit $\lambda \rightarrow 0^{-}$we are left with a harmonic oscillator. The curvatures of the minima and maxima tend to constant values as $\lambda \rightarrow 0^{-}$

$$
\begin{aligned}
V^{\prime \prime}(0) & =2(1-12 \lambda) \\
V^{\prime \prime}\left(x_{m}\right) & =\frac{8(36 \lambda+1+2 \sqrt{36 \lambda+1})}{3}=8+192 \lambda-864 \lambda^{2}+15552 \lambda^{3}+\cdots \\
V^{\prime \prime}\left(x_{M}\right) & =\frac{8(36 \lambda+1-2 \sqrt{36 \lambda+1})}{3}=-\frac{8}{3}+864 \lambda^{2}-15552 \lambda^{3}+\cdots
\end{aligned}
$$

Fig. 5 shows the potential-energy function of the oscillator (1) in the four regions just mentioned.

Fig. 4 shows that $E_{0}$ and $E_{1}$ remain isolated and become eigenvalues of the harmonic oscillator when $\lambda \rightarrow 0^{-}$. The reason is that they are below the minima of the side potentials. The eigenvalues $E_{2}, E_{3}$ and $E_{4}$ approach each other and become quasi degenerate for intermediate values of $\lambda$. As $\lambda \rightarrow 0^{-} E_{2}$ tends to a harmonic-oscillator eigenvalue while the pair $\left(E_{3}, E_{4}\right)$ remains quasi degenerate and moves upwards. When $E_{3}$ meets $E_{5}$ there is an avoided crossing after which $E_{3}$ approaches a harmonic oscillator eigenvalue while $E_{5}$ deviates upwards. The same situation takes place between $E_{4}$ and $E_{6}$, the former becomes a harmonic oscillator eigenvalue and the latter moves upwards. All the higher eigenvalues follow the same pattern; for example, $E_{4 k+1}, k=1,2, \ldots$, remain isolated till they are pushed upwards by a lower odd-parity eigenvalue. The eigenvalues $\left(E_{4 k+2}, E_{4 k+3}, E_{4 k+4}\right)$, $k=0,1, \ldots$, become quasi degenerate at intermediate values of $\lambda$ before the pair $\left(E_{4 k+3}, E_{4 k+4}\right)$ separates and moves upwards. It seems that every eigenvalue $E_{n}$ with $n>1$ undergoes an avoided crossing with a higher eigenvalue of the same symmetry before becoming a harmonic-oscillator eigenvalue. If $n>3$ the eigenvalue $E_{n}$ undergoes avoided crossings with $E_{n-2}$ and $E_{n+2}$ as illustrated in the more detailed Fig. 6 . The eigenvalues approach so closely that the avoided crossings appear actual crossings. We think that this chain of avoided crossings in which an eigenvalue changes slightly with $\lambda$ till it is pushed upwards by a lower eigenvalue of the same symmetry is perhaps the most interesting contribution of this paper since some of the other relevant features of the model have been discussed earlier.

In order to understand what happens at the avoided crossings we calculated $\Delta x=\sqrt{\left\langle x^{2}\right\rangle}$ for some states. This root-mean-square deviation is expected to be larger when the state is localized on the side wells. Fig. 7 show $\Delta x$ for the states with quantum numbers $n=0,2,4,6$. The states $n=0,2$ do not participate in avoided crossings and the corresponding $\Delta x$ does not change considerably as 

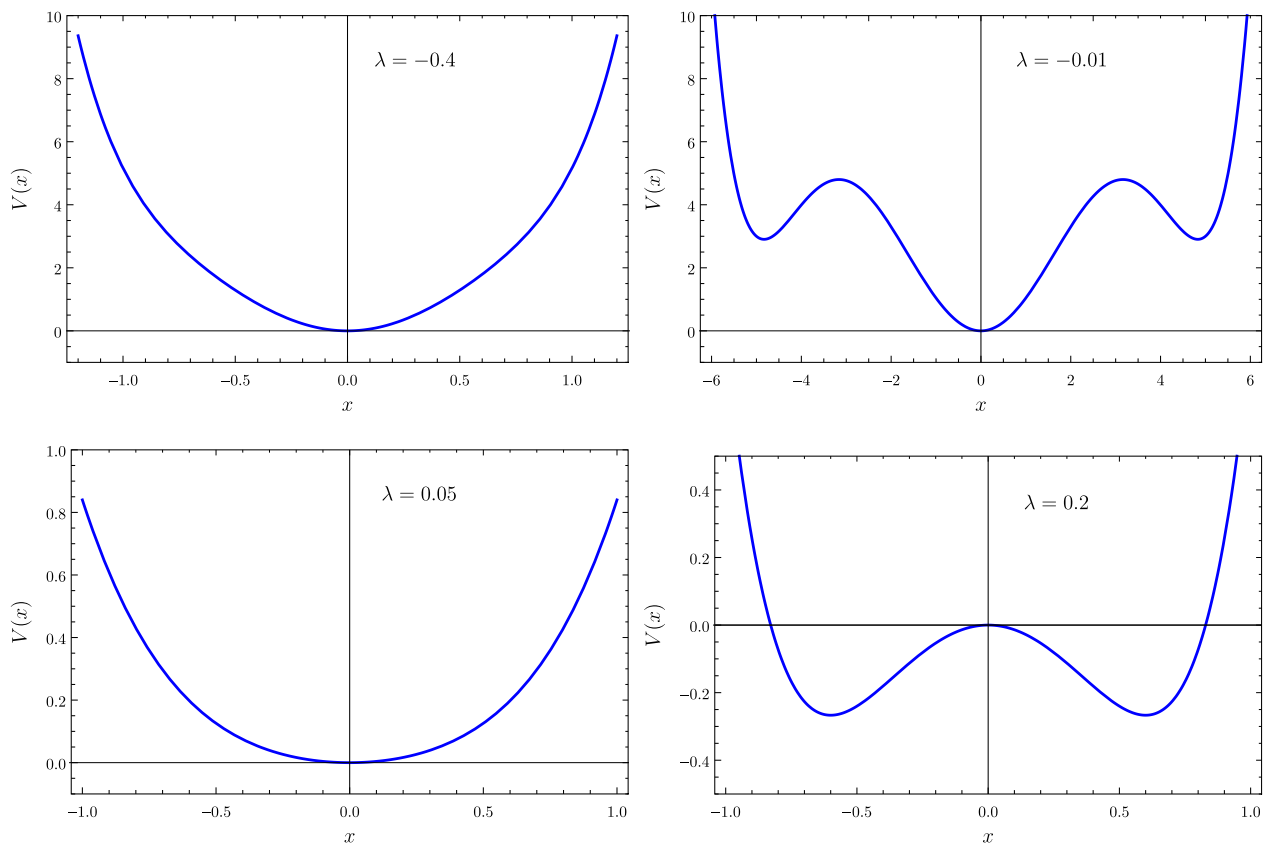

Fig. 5. Potential-energy function of the oscillator (1) in the four main regions of $\lambda$-values.

$\lambda \rightarrow 0^{-}$. The state $n=4$ undergoes an avoided crossing and $\Delta x$ exhibits a jump that suggests that it changes from being localized mainly on the central well to being localized mainly on the side ones. On the other hand, the state $n=6$ appears to be mainly localized on the side wells before the avoided crossing and mainly on the central one after it. In this case the jump is considerably larger indicating that the form of the eigenfunction changes more dramatically.

In closing this section we point out that the chain of avoided crossings just described may not be uncommon in the spectra of parameter-dependent Hamiltonians. In fact, one can easily construct a simple toy model with such properties. For example, the lowest eigenvalue of the matrix

$$
\mathbf{H}=\left(\begin{array}{lllll}
\lambda & 0.1 & 0 & 0 & 0 \\
0.1 & 1 & 0.4 & 0 & 0 \\
0 & 0.4 & 2 & 0.9 & 0 \\
0 & 0 & 0.9 & 3 & 1.6 \\
0 & 0 & 0 & 1.6 & 4
\end{array}\right)
$$

pushes the first excited state upwards that in turn pushes the next one upwards and so forth. The spectrum is shown in Fig. 8.

\section{Conclusions}

We revisited an old but interesting problem in quantum mechanics and mathematical physics. It has been our purpose to outline some remarkable features of its eigenvalues and eigenfunctions that have not been pointed out before. In particular, the spectrum for $\lambda<0$ exhibits a rich structure of avoided crossings. This chain of avoided crossings may be found in other quantum-mechanical problems as shown by the toy model (17). It occurs when one eigenvalue exhibits a positive slope larger than the ones of the excited states. It will then push the next excited state upwards provided that both belong to the same symmetry (otherwise an actual crossing takes place) and the process continues as long as there are excited states with smaller slope. At an avoided crossing the states 

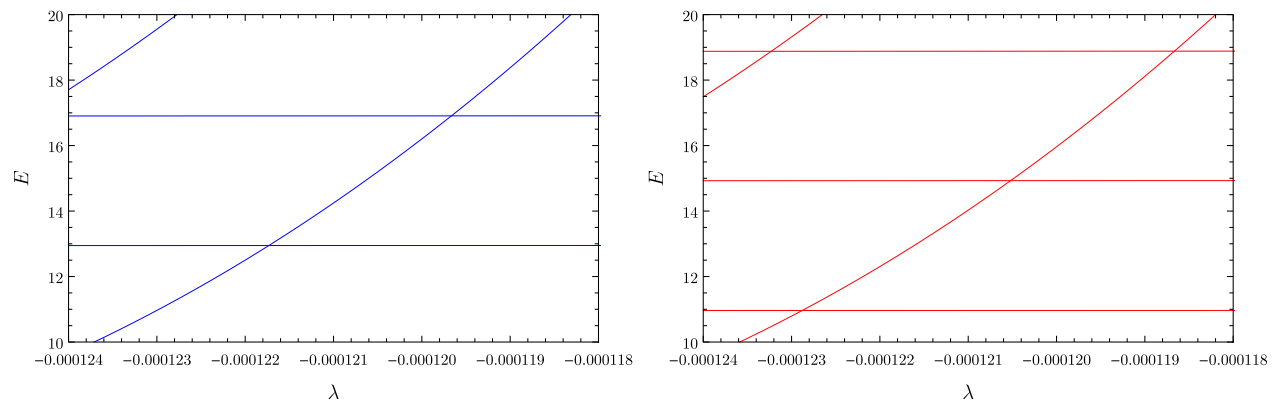

Fig. 6. Part of the spectrum of even (left) and odd (right) states of the anharmonic oscillator (1).
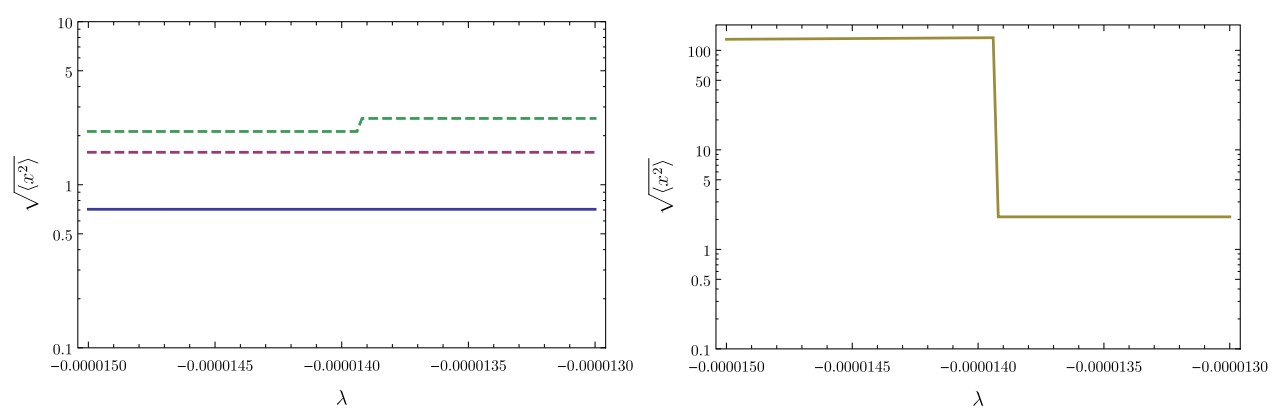

Fig. 7. $\sqrt{\left\langle x^{2}\right\rangle}$ for the states of the anharmonic oscillator (1) with quantum numbers $n=0,2,4$ (left) and $n=6$ (right).

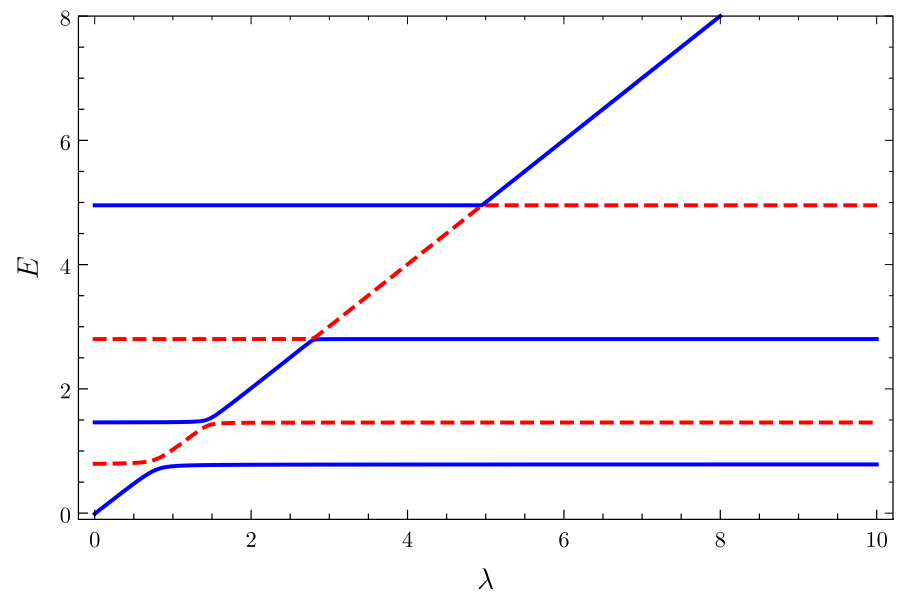

Fig. 8. Spectrum of the toy model (17).

that take part undergo dramatic changes in their form that are clearly revealed by the behaviour of the expectation value $\left\langle x^{2}\right\rangle(\lambda)$. We also estimated the asymptotic behaviour of the coefficients of the perturbation series and showed that they can be summed by means of Padé approximants and BorelPadé transformations for $\lambda>0$. This calculation was greatly facilitated by the hypervirial perturbation method that leads to straightforward recurrence relations for the perturbation corrections to the 
eigenvalues and expectation values $\left\langle x^{2 n}\right\rangle$ [3]. At present we do not know if there is any suitable approximation for $\lambda<0$. In this region we simply resorted to the Rayleigh-Ritz variational method with a basis set of 1000 eigenfunctions of the harmonic oscillator. The reason for such a large basis set is that the three widely separated wells pose a quite difficult problem for accurate calculation of the eigenfunctions and eigenvalues. We expect that present investigation may be a suitable complement to previous ones about this problem [1,2].

\section{References}

[1] I.W. Herbst, B. Simon, Phys. Lett. B 78 (1978) 304-306; Phys. Lett. B 80 (1979) 433 (erratum).

[2] A.V. Turbiner, Phys. Rep. 642 (2016) 1-71.

[3] F.M. Fernández, Introduction To Perturbation Theory in Quantum Mechanics, CRC Press, Boca Raton, 2001.

[4] J. Zinn-Justin, Ann. Inst. Fourier (Grenoble) 54 (2003) 1259-1285.

[5] C.M. Bender, Phys. Rev. 184 (1969) 1231-1260.

[6] B. Simon, A. Dicke, Ann. Phys. 58 (1970) 76-136. 